\title{
Hiperplasia fibrosa inflamatória: relato de caso e revisão de literatura
}

\author{
Antônio Fernando Pereira Falcão ${ }^{1}$ \\ Patricia Leite Ribeiro Lamberti ${ }^{2}$ \\ Felipe da Gama Lobo Lorens ${ }^{3}$ \\ Juliana Andrade de Lacerda ${ }^{4}$ \\ Bruno Campos Nascimento ${ }^{4}$
}

\section{Resumo}

A hiperplasia fibrosa inflamatória, também denominada de epúlide fissurada, enquadra-se entre as lesões benignas do tecido mole decorrentes de traumas crônicos, geralmente de uma prótese mal adaptada. Essa lesão acomete preferencialmente o sexo feminino, adultos de meia idade ou mais velhos, e usuários de próteses. É mais frequente na região anterior da maxila, exofítica, e pode variar de flácida a consistente. O presente trabalho relata um caso de hiperplasia fibrosa inflamatória, diagnosticado em uma mulher de cinquenta dois anos de idade, leucoderma, no ambulatório de Estomatologia I da Faculdade de Odontologia da Universidade Federal da Bahia, e apresenta relatos da literatura a respeito dessa lesão.

Palavras-chave: Hiperplasia fibrosa inflamatória; Epúlide fissurada; Próteses mal adaptadas - Lesōes.

\section{INTRODUÇÃO E REVISÃO DE LITERATURA}

A hiperplasia fibrosa inflamatória é uma lesão proveniente de uma reação hiperplásica do tecido conjuntivo fibroso (SAPP; EVERSOLE; WYSOCKI, 1997). Essa proliferação benigna ocorre em resposta a injúrias crônicas de baixa intensidade (SANTOS; COSTA; SILVA NETO, 2004).

Outras sinonímias para essa lesão foram apresentadas por Neville e colaboradores (2004), como epúlide fissurada, tumor por lesão de dentadura, epúlide por dentadura, sendo referida também por Alves e Gonçalves (2005) como hiperplasia fibrosa traumática. Contudo, ela é denominada melhor como hiperplasia fibrosa inflamatória (NEVILLE et al., 2004; SANTOS; COSTA; SILVA NETO, 2004).

Cruz (2005) constatou que, dentre os 295 casos submetidos à biópsia, a lesão mais frequente foi a hiperplasia fibrosa inflamatória, o que está de acordo com o levantamento epidemiológico realizado por Torreão e colaboradores (1999).

\footnotetext{
${ }^{1}$ Professor Associado do Departamento de Propedêutica e Clínica Integrada- Faculdade de Odontologia - UFBA. Salvador.

${ }^{2}$ Doutora em Radiologia Odontológica UFBA/UFPB; Professora Adjunta- Faculdade de Odontologia da UFBA.

${ }^{3}$ Especialista em Radiologia.-Faculdade de Odontologia da UFBA; Professor de Radiologia e Estomatologia- Faculdade de Odontologia UFBA.

${ }^{4}$ Acadêmicos de Odontologia da UFBA.
}

Correspondência para / Correspondence to:

Antônio Fernando Pereira Falcão.

Departamento de Estomatologia I - Faculdade de Odontologia -

Universidade Federal da Bahia.

Av. Araújo Pinho, 62 - Canela.

40110-912. Salvador - Bahia - Brasil.

Tel: (71) 3283-8964.

E-mail: afpfalcao@hotmail.com 
Há uma frequente relação entre o aparecimento da hiperplasia fibrosa inflamatória e a utilização de próteses mal adaptadas (ALVES; GONÇALVES, 2005; COELHO; SOUSA; DARÉ, 2004; NEVILLE et al., 2004; SAPP; EVERSOLE; WYSOCKI, 1997). Coelho, Sousa e Daré (2004) relatam que o aumento do período de uso das próteses está associado com o aumento da hiperplasia fibrosa inflamatória, sugerindo que prótese totais e parciais removíveis, mal adaptadas e (ou) antigas, geralmente ocasionam traumas constantes e inflamação dos tecidos orais. Segundo Alves e Gonçalves (2005) e Coelho, Sousa e Daré (2004), o aparecimento da lesão em próteses novas pode ocorrer em resposta à pressão exercida pelos bordos cortantes da prótese no vestíbulo anterior.

Além da influência de próteses mal adaptadas no aparecimento dessa lesão, enquadram-se, como fatores etiológicos, diastemas, bordos cortantes de dentes, procedimentos iatrogênicos e higiene bucal inadequada (BASSI; VIEIRA; GABRIELLI, 1998; SANTOS; COSTA; SILVA NETO, 2004).

O uso de próteses totais imediatas, através da reabsorção óssea da crista alveolar e da cicatrização do alvéolo, que ocorrem após o processo cirúrgico, pode provocar a desadaptação da prótese, ocasionado, assim, a hiperplasia fibrosa inflamatória (ALVES; GONÇALVES, 2005).

A hiperplasia fibrosa inflamatória pode advir de próteses confeccionadas com câmara de sucção, uma depressão central com bordos afiados (BORAKS, 1996), ressaltando-se que, atualmente, sua confecção é condenada (FRANÇA; SOUZA, 2003). O surgimento da lesão é proveniente do mecanismo de sucção da mucosa do palato, como uma forma de melhor adaptação do aparelho protético pelo organismo (BARBOSA et al., 1988 apud FRANÇA; SOUZA, 2003).

A hiperplasia fibrosa inflamatória é mais prevalente no sexo feminino, de acordo com a maioria dos estudos, compreendendo dois terços a três quartos dos casos biopsiados. Essa lesão acomete principalmente a região anterior da maxila e mandíbula em relação à região posterior
(NEVILLE et al., 2004); segundo o estudo realizado por Alves e Gonçalves (2005), a região predominante é o sulco vestibular.

Alves e Gonçalves (2005) encontraram casos de hiperplasia fibrosa inflamatória em todas as faixas etárias, com prevalência na quinta década de vida. Observando a variável raça, a concentração de ocorrências foi soberana em indivíduos leucodermas, em relação aos xantodermas. Segundo estudos de Torreão e colaboradores (1999), houve predominância pela sexta década de vida, seguida pela quarta e quinta décadas.

A HFI pode se apresentar como um processo exofítico, ou como uma placa bem definida. Considerando a consistência, a lesão pode variar de firme a flácida quando submetida à palpação. A base pode ser séssil ou pediculada, com coloração semelhante à mucosa ou eritematosa, de crescimento lento, sendo geralmente assintomática (SANTOS; COSTA; SILVA NETO, 2004). A superfície caracterizase como lisa, podendo se apresentar como um molde negativo da câmara de sucção. Essa também pode apresentar superfície vegetante, semelhante à hiperplasia papilar, a qual pode estar associada à hiperplasia fibrosa inflamatória (FRANÇA; SOUZA, 2003). Comumente, a prótese adapta-se entre as pregas de tecido hiperplásico no vestíbulo alveolar (NEVILLE et al., 2004).

A lesão apresenta as seguintes características histopatológigas: epitélio pavimentoso estratificado, que envolve tecido conjuntivo fibroso hiperplásico, com número elevado de fibras colágenas, apresentando também alto grau de células inflamatórias crônicas e quantidade variável de vasos sanguíneos, sendo que o epitélio pode ser ceratinizado ou não (BASSI; VIEIRA; GABRIELLI, 1998; COUTINHO; SANTOS, 1998). De acordo com Neville e colaboradores (2004), áreas ulceradas não são incomuns nas fissuras entre as pregas. Neville e colaboradores (2004), e Sapp, Eversole e Wysocki (1997) ressaltam também que, quando há o envolvimento de glândulas salivares menores na lesão, pode-se observar usualmente sialodenite crônica. 
A HFI pode estar relacionada a infecções fúngicas, devido ao uso inadequado de próteses (BASSI; VIEIRA; GABRIELLI, 1998). De acordo com Moreira e colaboradores (2002), o uso de prótese é um fator predisponente ao surgimento dessas infecções. A candidíase associada à prótese é um tipo de candidose eritematosa, sendo referida por candidíase atrófica crônica ou estomatite por dentadura. Clinicamente, apresenta-se como uma região eritematosa, que pode estar acompanhada por petéquias hemorrágicas nas bordas de dentaduras de uma prótese superior removível. Para o tratamento das infecções fúngicas, o uso de Nistatina e Itraconazol, na forma de suspensão oral e solução oral, respectivamente, é indicado (NEVILLE et al., 2004).

$O$ procedimento terapêutico indicado para o tratamento da HFI é a remoção cirúrgica da lesão (NEVILLE et al., 2004; SANTOS; COSTA; SILVA NETO, 2004). Outras terapêuticas, sugeridas por Santos, Costa e Silva Neto (2004), que podem ser adotadas em determinados casos, são a microabrasão, o uso do laser ou a crioterapia. A realização da biópsia é importante para confirmar o diagnóstico de hiperplasia fibrosa inflamatória, visto que essa lesão faz diagnóstico diferencial com lipofibroma, neurofibroma, rabdomioma, leiomioma e tumores de glândulas salivares menores (COUTINHO; SANTOS, 1998) e também com o granuloma piogênico e o fibroma ossificante periférico (COLEMAN; NELSON, 1996).

O sucesso do tratamento depende também da correção da prótese mal adaptada, ou confecção de uma nova prótese, quando esse é o fator etiológico (NEVILLE et al., 2004; SANTOS; COSTA; SILVA NETO, 2004). Acrescenta-se que, de acordo com Castro e colaboradores (1995) e Bassi, Vieira e Gabrielli (1998), é possível haver regressão de lesões iniciais pouco elevadas com a remoção da prótese. Além da intervenção protética, orientaçôes a respeito da higiene oral e da prótese são indispensáveis (COELHO; SOUSA; DARÉ, 2004).

Tendo em vista a associação do uso de próteses mal adaptadas com a ocorrência de lesões orais, como a hiperplasia fibrosa inflamatória, está sob a responsabilidade do cirurgião-dentista diagnosticar o aparecimento de manifestações orais, assim como preveni-las, orientando os usuários de próteses sobre a sua adequada higienização e a necessidade da substituição (ALVES; GONÇALVES, 2005; SANTOS; COSTA; SILVA NETO, 2004).

Este trabalho contempla uma revisão de literatura sobre o assunto em questão, a hiperplasia fibrosa inflamatória, destacando suas características clínicas, os fatores etiológicos, a histopatologia da lesão e o tratamento, assim como, o relato de um caso clínico da referida patologia.

\section{RELATO DO CASO CLÍNICO}

Paciente M.A.S.S., 52 anos, do sexo feminino, leucoderma, dona de casa, natural de Simão Dias (SE), casada procurou a disciplina de Estomatologia I da Faculdade de Odontologia da Universidade Federal da Bahia.

A paciente relatou crescimento da mucosa na região de fundo de vestíbulo anterior da maxila, havendo sintomatologia dolorosa ao toque nesse local e, ocasionalmente, na região de asa do nariz, com aumento de volume e da intensidade da dor esporadicamente. Para cessar a dor, a paciente fez uso de anti-inflamatórios não esteróides, como diclofenaco de sódio, apesar de apresentar hipersensibilidade a esses medicamentos. Referiu-se também à sensação de prurido e alteração de cor nos lábios.

A respeito da história médicoodontológica, constatou-se que a paciente é hipertensa, faz uso do medicamento besilato de anlodipina, apresenta gastrite, cefaléias com frequência e, ocasionalmente, dor no peitoral. Apresentou Herpes zoster e Síndrome do pânico. Teve acesso a serviços odontológicos nos dois meses anteriores à presente consulta. Faz uso de uma prótese parcial superior removível há aproximadamente dez anos.

Os sinais vitais constatados foram: pressão arterial 160/90 mmHg, frequência cardíaca 40 b.p.m e frequência respiratória 16 i.p.m . Não é fumante, não faz uso de bebidas alcoólicas, e tem um bom relacionamento social. Quanto aos 
hábitos parafuncionais, apresenta bruxismo. Atualmente, faz escovação três vezes ao dia, porém antes não realizava uma boa higiene bucal.

No exame físico extrabucal, foi observada a presença de queilite angular na comissura labial direita. $\mathrm{Na}$ inspeção do pescoço e na palpação dos gânglios, não foram identificadas alterações dignas de nota. Ao realizar a palpação da ATM, a paciente revelou ausência de sintomatologia dolorosa, e não foram percebidos deslocamentos, estalidos ou crepitaçôes durante os movimentos mandibulares.

Ao exame físico intrabucal, foi identificada uma lesão exofítica, na região de fundo de vestíbulo anterior da maxila, de consistência flácida, superfície lisa, de coloração semelhante à mucosa, de forma arredondada, de inserção pediculada, ocasionalmente sintomática, e com dois centímetros de extensão (Figura 1; Figura 2).

Foi evidenciada também a presença de candidíase atrófica no palato duro, causada pela prótese mal adaptada e hábitos de higiene oral inadequados.

Foram solicitadas radiografias periapical do primeiro molar superior direito, que apresentava fratura e oclusal total da maxila. Os aspectos radiográficos da maxila estavam dentro do padrão de normalidade. A radiografia oclusal demonstrou ausência de envolvimento ósseo na regiāo afetada.

O diagnóstico clínico estabelecido foi de hiperplasia fibrosa inflamatória, candidíase atrófica crônica, queilite angular e gengivite.

A terapêutica instituída para a candídiase atrófica crônica consistiu no uso de antifúngico durante quinze dias. Foi prescrito o medicamento Nistatina, suspensão oral, recomendando-se à paciente o bochecho do produto e posterior deglutição, nos primeiros sete dias, e, nos dias seguintes, o produto deveria ser desprezado após o bochecho. Após o período, observaram-se focos remanescentes da candidíase e, com isso, foi prescrita uma nova sessão terapêutica, utilizando-se o mesmo medicamento e recomendações.

A paciente foi encaminhada para a disciplina de Estomatologia II, onde se realizou a biopsia excisional (Figura 3; Figura 4). Após a remoção cirúrgica da lesão, foi realizado o exame histopatológico. Através desse, observou-se uma camada de epitélio pavimentoso estratificado com acantose, paraqueratinizado, com projeções epiteliais em direção ao tecido conjuntivo, o qual apresentava grossos feixes de fibras colágenas com fibroblastos, vasos sanguíneos e um infiltrado inflamatório crônico, confirmando assim, o diagnóstico de hiperplasia fibrosa inflamatória (Figura 5; Figura 6).

No pós-operatório, observou-se um ótimo processo de reparo tecidual (FIGURA 7). Além dessas terapêuticas instituídas, foi recomendada a não-utilização da prótese antiga, sendo a paciente encaminhada, após a regressão da candidíase, para a confecção de uma nova prótese, bem como para o serviço de urgência da faculdade para a exodontia da unidade dentária fraturada.

\section{DISCUSSÃO}

Os achados clínicos referidos no caso clínico relatado condizem com a maioria dos dados apresentados na literatura. As características clínicas correspondem a um quadro típico de hiperplasia fibrosa inflamatória, visto que ela é geralmente localizada na região anterior da maxila e de cor semelhante à mucosa, exofítica, de crescimento lento, podendo ser pediculada. (BASSI; VIEIRA; GABRIELLI, 1998; COUTINHO; SANTOS, 1998; SANTOS; COSTA; SILVA NETO, 2004; NEVILLE et al., 2004).

A lesão acomete preferencialmente indivíduos do sexo feminino, de meia idade ou mais velhos e leucodermas, segundo Alves e Gonçalves (2005) e Neville e colaboradores (2004), apresentando o paciente, acompanhado no ambulatório de Estomatologia I da FOUFBA, esse perfil. Discordando das descrições de Coutinho e Santos (1998), de Neville e colaboradores (2004) e de Santos, Costa e Silva Neto (2004), o caso clínico apresenta uma lesão ocasionalmente sintomática.

Há um consenso em relação ao fator predisponente mais associado ao aparecimento da lesão, dentre as possíveis etiopatogenias. Alves e Gonçalves (2005), Bassi, Vieira e Gabrielli (1998), Neville e colaboradores (2004), Sapp, 
Figura 1. Lesão adaptada ao bordo da prótese.

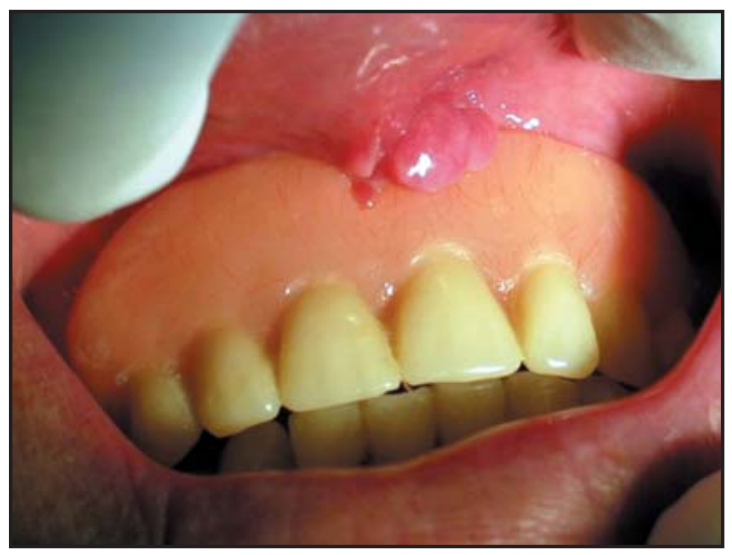

Figura 2. Aspecto clínico da lesão.

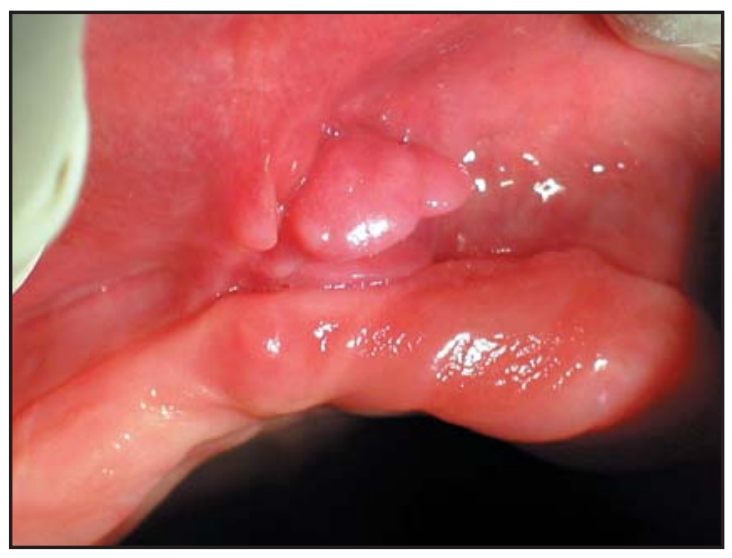

Figura 3. No momento da excisão cirúrgica.

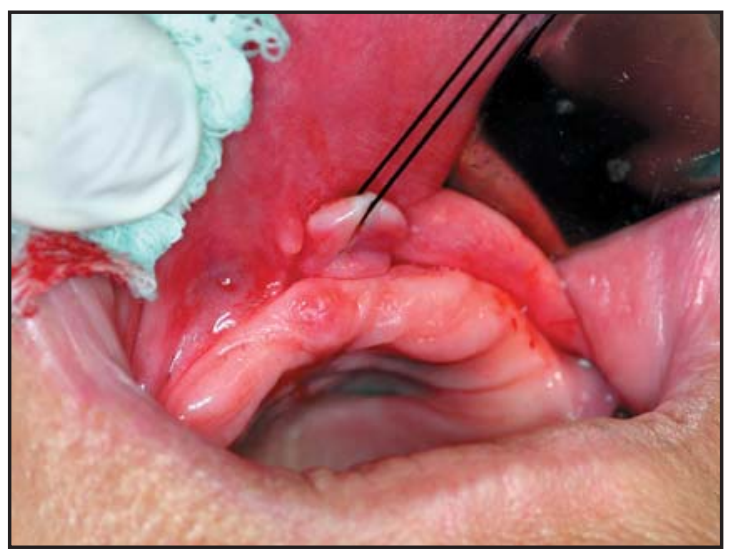

Eversole e Wysocki (1997) defendem a estreita relação com o trauma crônico de baixa intensidade, provocado por próteses mal adaptadas na mucosa oral, com o surgimento da hiperplasia fibrosa inflamatória.

Segundo França e Souza (2003), e Moreira e colaboradores (2002), a ocorrência
Figura 4. Após a excisão cirúrgica.

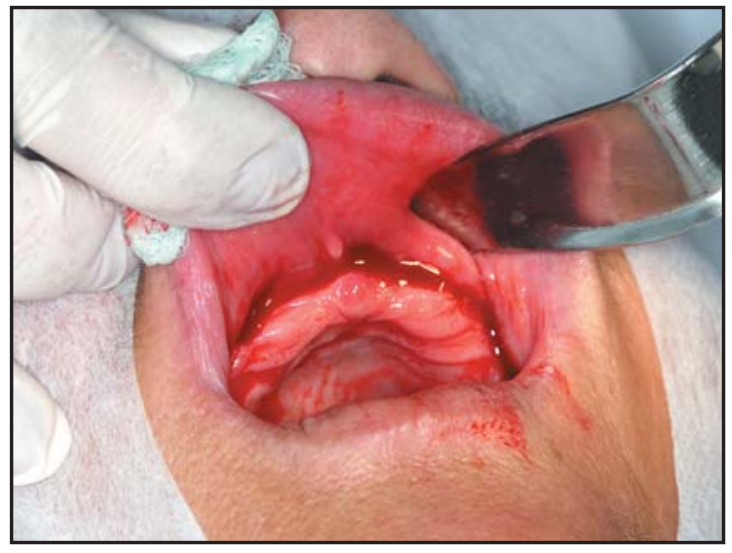

Figura 5. Aspectos histológicos da lesão.

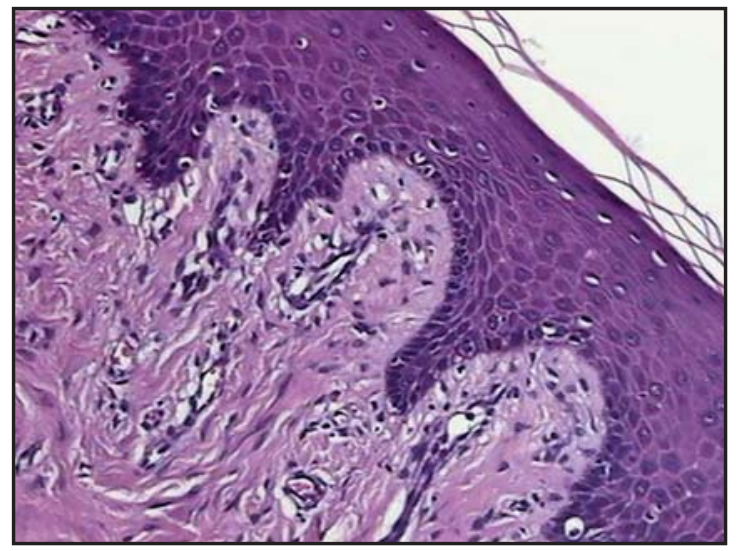

Figura 6. Aspectos histológicos da lesão.

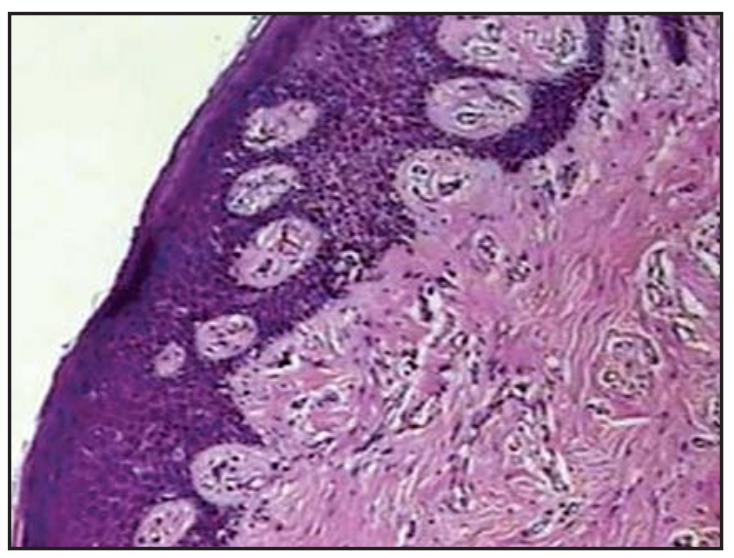

de estomatite protética está relacionada ao uso de prótese, pelo fato de a higiene oral e da prótese ser insatisfatória, bem como o seu uso contínuo (FRANÇA; SOUZA, 2003), o que é evidenciado no caso clínico relatado.

Neville e colaboradores (2004) relata que a hiperplasia papilar inflamatória pode surgir 
Figura 7. Aspecto clínico após proservação de 1 mês.

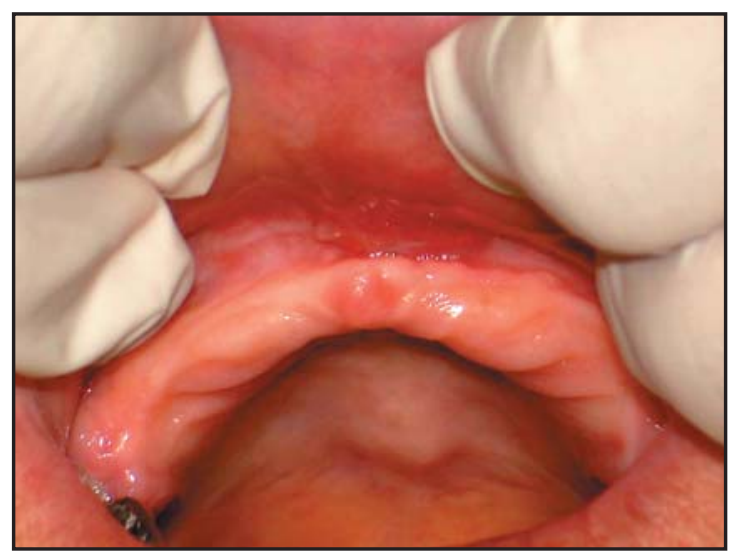

associada à superfície da hiperplasia fibrosa inflamatória esporadicamente. Entretanto, segundo Reichart e colaboradores (1994 apud TEÓFILO, 2003 ), a hiperplasia papilomatosa inflamatória pode ser considerada como um tipo de hiperplasia fibrosa inflamatória. No caso clínico apresentado, a paciente não apresentava hiperplasia papilar inflamatória.

Os exames radiográficos não revelaram envolvimento ósseo, indicando, assim, uma lesão de tecido mole, o que está de acordo com o que foi apontado do por Coutinho e Santos (1998).

O estudo anatomo-patológico da lesão do caso clínico e os dados da literatura caracterizam, histologicamente, a hiperplasia fibrosa inflamatória como um estroma de tecido conjuntivo fibroso, com um infiltrado de células inflamatórias crônicas, havendo a presença de vasos e um epitélio pavimentoso estratificado, hiperplásico, ceratinizado (NEVILLE et al., 2004; SAPP; EVERSOLE; WYSOCKI, 1997).

Diversos autores, como Neville e colaboradores (2004), Coutinho e Santos (1998), Santiago, Gusmão e Silva (2003), estabelecem como terapêutica de eleição a biópsia excisional. Aliado a essa terapêutica, recomenda-se o reajuste ou a confecção de uma nova prótese, assim como orientações referentes à higiene oral e protética (COELHO; SOUZA; DARÉ, 2004). O plano de tratamento do caso clinico incluiu a opção terapêutica e as orientaçôes supracitadas.

Coelho, Souza e Daré (2004) consideram o prognóstico excelente, o que está de acordo com o paciente em acompanhamento.

\section{CONSIDERAÇÕES FINAIS}

A hiperplasia fibrosa inflamatória é uma lesão proliferativa de tecidos mole, não neoplásica, prevalente na cavidade oral.

Acomete mais frequentemente indivíduos do sexo feminino e leucodermas que fazem uso de próteses mal adaptadas. É importante também estar atento para outros fatores traumáticos que podem desencadear a lesão.

Para o sucesso terapêutico, é imprescindível, além da remoção cirúrgica, a eliminação do agente traumático.

É necessário que o cirurgião-dentista esteja apto a fazer um correto diagnóstico e instituir um tratamento eficiente, assim como confeccionar e orientar adequadamente o uso de próteses.

\title{
Inflammatory fibrous hyperplasia: a case report and literature review
}

\begin{abstract}
Inflammatory fibrous hyperplasia, also denominated by epulis fissuratum, is related to benign lesions of soft tissue caused by chronic traumas, usually of a prosthesis badly adapted. This lesion attacks the feminine sex preferentially, adults of middle age or older, and users of prostheses. It is more frequent in the anterior area of the jawbone, exophytic, and it can vary from flaccid to solid. The present work reports a case of inflammatory fibrous hyperplasia diagnosticated in a fifty two years old white woman in the clinic of Estomatology I of University of Odontology of UFBA, and it presents reports of the literature regarding that lesion.
\end{abstract}

Keywords: Inflammatory fibrous hyperplasia; Epulis fissuratum; Prosthesis badly adaptedLesions. 


\section{REFERÊNCIAS}

ALVES, N.C.; GONÇALVES, H.H.S.B. Estudo descritivo da ocorrência de hiperplasias fibrosas inflamatórias observadas no Serviço do Laboratório de Histopatologia Bucal da Faculdade de Odontologia de Marília. R. Paul. Odontol., São Paulo, n.4, p.4-8, out./dez. 2005.

BASSI, A.P.F.; VIEIRA, E.H.; GABRIELLI, M.A.C. Hiperplasia fibrosa inflamatória. RGO, Porto Alegre, v.4, n.46, p.209-211, out./dez. 1998.

BORAKS, S. Diagnóstico bucal. São Paulo: Artes Médicas, 1996.

BRITO, A.M. de; VELOSO, K.M.M. Lesões causadas por próteses totais mal adaptadas em pacientes idosos: relato de casos clínicos. 2006. Disponível em: <http:// w w w. od o n t o log i a c o m . b r / artigos.asp? $\mathrm{id}=189 \&$ idesp $=37 \&$ ler $=$ s $>$. Acesso em: 27 maio 2007.

CASTRO, A.L. et al. Estomatologia. 2.ed. São Paulo: Santos, 1995.

COELHO, C.M.; SOUSA, Y.T.; DARÉ, A.M. Denture-related oral mucosal lesions in a Brazilian school of dentistry. J. Oral Rehabil., Oxford, v.31, p.135-139, 2004.

COLEMAN, G.C.; NELSON, J.F. Princípios de diagnóstico bucal. Rio de Janeiro: Guanabara Koogan, 1996.

COUTINHO, T.C.L.; SANTOS, M.E.O. Hiperplasia fibrosa inflamatória. RGO, Porto Alegre, v.1, n.46, p.27-34, jan./mar. 1998.

CRUZ, M.C. Levantamento das biópsias da cavidade oral realizadas no hospital universitário - Unidade Presidente Dutra/ UFMA, da cidade de Sâo Luís MA, no período de 1992 a 2002. R. Bras. Patol. Oral, Natal, v.4, n.3, p.185-188, jul./set. 2005.
FRANÇA, B.H.S.; SOUZA, A.M. Prevalência de manifestaçōes estomatológicas originárias do uso de próteses totais. JBC: J. Bras. Clin. Odontol. Integrada, Curitiba, v.40, n.7, p.296300, jul./ago. 2003.

MOREIRA, A.C.A. et al. Isolamento de Candida parapslosis em pacientes com diagnóstico clínico de candidíase atrófica crônica. R. Ci. Méd. Biol., Salvador, v.1, n.1, p.124-128, nov. 2002.

NEVILLE, B.W. et al. Patologia oral e maxilofacial. 2.ed. Rio de Janeiro: Guanabara Koogan, 2004.

SANTIAGO, L.M.; GUSMÃO, E.S.; SILVA, U.H. Fibroma ossificante periférico e hiperplasia fibrosa inflamatória: relato de caso clínico. Odontol. Clín.-cient., Recife, v.2, n.3, p.233240, set./dez. 2003.

SANTOS, M.E.S.M.; COSTA, W.R.M.; SILVA NETO, J.C. Terapêutica cirúrgica da hiperplasia fibrosa inflamatória: relato de caso. R. Cir. Traumatol. Buco-MaxiloFac., Recife, v.4, n.4, p.241-245, out./dez. 2004.

SAPP, J.P; EVERSOLE, L.R; WYSOCKI, G.W. Contemporary oral and maxilofacial pathology. St. Louis: Mosby, 1997.

TEÓFILO, J.M. Patologia oral: resumo didático: lesões reacionais de interesse

odontológico. J. Odondol. FAFICA, Catanduva, v.3, n.1, 2003.

TORREÃO, A.C.R. et al. Levantamento epidemiológico de biópsias da regiāo buçomaxilo-facial encaminhadas ao Laboratório de Patologia Bucal da Faculdade de Odontologia de Pernambuco. R. Cons. Reg. Odontol. Pernamb., Recife, v.2, n.2, p.118-125, out. 1999. 